\title{
Simultaneous Upper and Lower Jaw Extractions, Autogenous Bone Augmentation and Temporary Implant Placement with Immediate Loading
}

\author{
Philippe Van Camp, Geert Klomp, Erik Nout \\ Department of Oral and Maxillofacial Surgery, Elisabeth-Tweesteden Hospital, Tilburg, The Netherlands \\ Email: philippe.vcthk@gmail.com
}

How to cite this paper: Van Camp, P., Klomp, G. and Nout, E. (2020) Simultaneous Upper and Lower Jaw Extractions, Autogenous Bone Augmentation and Temporary Implant Placement with Immediate Loading. Open Journal of Stomatology, 10, 383-395.

https://doi.org/10.4236/ojst.2020.1012035

Received: November 17, 2020

Accepted: December 25, 2020

Published: December 28, 2020

Copyright $\odot 2020$ by author(s) and Scientific Research Publishing Inc. This work is licensed under the Creative Commons Attribution International License (CC BY 4.0).

http://creativecommons.org/licenses/by/4.0/

\section{(cc) (i) Open Access}

\begin{abstract}
Aims: We expanded the known technique for simultaneously augmenting an atrophic maxilla and placement of immediate provisional implants (IPI), followed by immediate loading by performing surgery in both jaws simultaneously. Feasibility of this new technique, implant survival and success were evaluated as well as prosthetic success. Materials and Methods: All patients undergoing simultaneous bone grafting and IPI placement with immediate loading at our institute between the 1st of June 2016 and the 30th of May 2018 were included and followed up for at least one year postoperatively. Results: 3 patients were followed for a mean period of 25.67 months (20 - 29 months). 33 IPIs were placed. All were immobile at second stage surgery without signs of infection. No provisional bridges were lost and no infections were noted. After second stage surgery, none of these 36 final dental implants were lost. There was some bone loss at one implant. In all patients, good functional and aesthetic results were obtained without any unforeseen complications. This renders the implant survival at $100 \%$ and the success rate at $97 \%$. Conclusion: The technique is complex due to the intricate step-by-step process that is required and depends on a dedicated team to ensure a proper workflow. When performed correctly, the protocol shows good and predictable results.
\end{abstract}

\section{Keywords}

Dental Implant Surgery, Immediate Dental Implant Loading, Implant-Supported Dental Prosthesis, Bone Transplantation

\section{Introduction}

Many edentulous patients suffer from limited or no retention of their removable 
prosthesis in upper and/or lower jaw, which severely hinders masticatory function and patient comfort as well as general quality of life. The use of endosseous dental implants is a well-established solution for these patients, provided sufficient bone volumes are present for implantation. Furthermore, patients with terminally diseased dentition might have the same desire for a fixed rehabilitation option.

In patients where the bony structures do not allow implant placement, alveolar ridge augmentation is indicated prior to the placement of dental implants. Traditionally, augmentation of the alveolar ridge in the edentulous maxilla is performed by buccal plating and/or a bilateral sinus lift procedure. In the mandible merely buccal plating and/or guided tissue regeneration techniques are conducted. The standard treatment protocol after bone grafting prescribes a healing period of $4-6$ months to permit graft healing, after which implants can be placed. Furthermore, an osseointegration period of 3 months is maintained if immediate loading is not feasible or desired. In dentate cases requiring removal of all remaining teeth, an additional period of 3 months needs to be added to account for the expected atrophy following extraction. Patient compliance is higher when one does not need to cope with ill-fitting prostheses and social discomfort during these rather long healing periods. Provisional prostheses also attribute to an increased resorption of the graft and poor healing due to the exerted pressure on the surgical area [1] [2]. To overcome these issues, meet patient's prosthetic wishes and improve satisfaction rates, Lenssen et al. [1] came up with a technique providing a fixed prosthesis immediately postoperatively after augmentation of a severely atrophic maxilla. This treatment method was further developed by Barbier and colleagues from the same institute [3]. In our institute, we also achieved good and predictable results in dentulous cases as well as in edentulous single jaw cases utilizing this technique. In this report we would like to share our experience and difficulties encountered when applying this technique in double jaw cases. In addition, we propose some adaptations to the previously reported procedures, which we think are beneficial for patient and surgeon alike. To the best of our knowledge, these adaptations have not been previously reported.

\section{Materials and Methods}

This study was prepared using the CARE guidelines [4] and is in accordance with the Helsinki Declaration of 1975 (revised in 2000).

\subsection{Patients}

All patients undergoing simultaneous bone grafting and IPI placement (6 in the upper jaw and 5 or 6 in the lower jaw) with immediate loading at our institute between the 1st of June 2016 and the 30th of May 2018 were included and followed up for at least one year postoperatively. There was no age limit applied in this study. Patients undergoing this procedure in a single jaw and patients where 
a different type of implant was used, were excluded. The final exclusion criterion was failure of the patient to complete routine check-ups up to one year postoperatively. Patient data were retrieved from the patient files. The inpatient files and the notes during outpatient visits were scrutinised for signs of infection. IPI survival and implant survival were defined as the IPI or implant still being present intraorally without signs of infection or clinical mobility. The implant success criteria by Albrektsson [5] are still the most widely used [6]. However, Papspyridakos et al. [7] in their study correctly note the lack of criteria regarding the success at prosthetic level. Because of this reason we scored our final restoration success as a combination of signs of infection and restorative or technical complications. Patient satisfaction was also taken into account. See Table 1 for details.

\subsection{Adapted Technique}

The suitable patient is edentulous with insufficient bone volumes (Cawood and Howell [8] class IV to VI) to allow implant placement or has a terminal dentition with a high aesthetic wish and a strong desire to avoid wearing removable prostheses. After proper diagnosis including radiologic imaging, a treatment plan is devised. The patient receives extensive information explaining all necessary steps, procedures, complications and associated costs. After obtaining an informed consent, the process is initiated. Figure 1 shows a flowchart of the complete process. In the prosthodontic preparation phase, casts of the upper and lower jaw are fabricated by means of an intraoral scan (Trios 3, 3Shape, Copenhagen, Denmark). The data is sent to the dental lab where a provisional setup is made in close communication with the prosthodontist. Clear collaboration between dentist and dental lab is of utmost importance regarding occlusion, vertical relation between upper and lower jaw, teeth alignment and aesthetics. All of

Table 1. Applied implant and restorative success criteria.

\begin{tabular}{|c|c|}
\hline Main level & Subset of details judged \\
\hline \multirow{5}{*}{ Implant level } & Mobility \\
\hline & Pain \\
\hline & Radiolucency \\
\hline & Infection \\
\hline & Bone loss $>1.5 \mathrm{~mm}$ after the first year in function \\
\hline \multirow{4}{*}{ Peri-implant soft-tissue level } & Suppuration \\
\hline & Bleeding \\
\hline & Swelling \\
\hline & Probing depth $>3 \mathrm{~mm}$ \\
\hline \multirow{3}{*}{ Prosthetic level } & Adequate masticatory function \\
\hline & Complications \\
\hline & Adequate maintenance \\
\hline \multirow{3}{*}{ Patient satisfaction level } & Esthetics \\
\hline & Comfort \\
\hline & Overall patient satisfaction \\
\hline
\end{tabular}




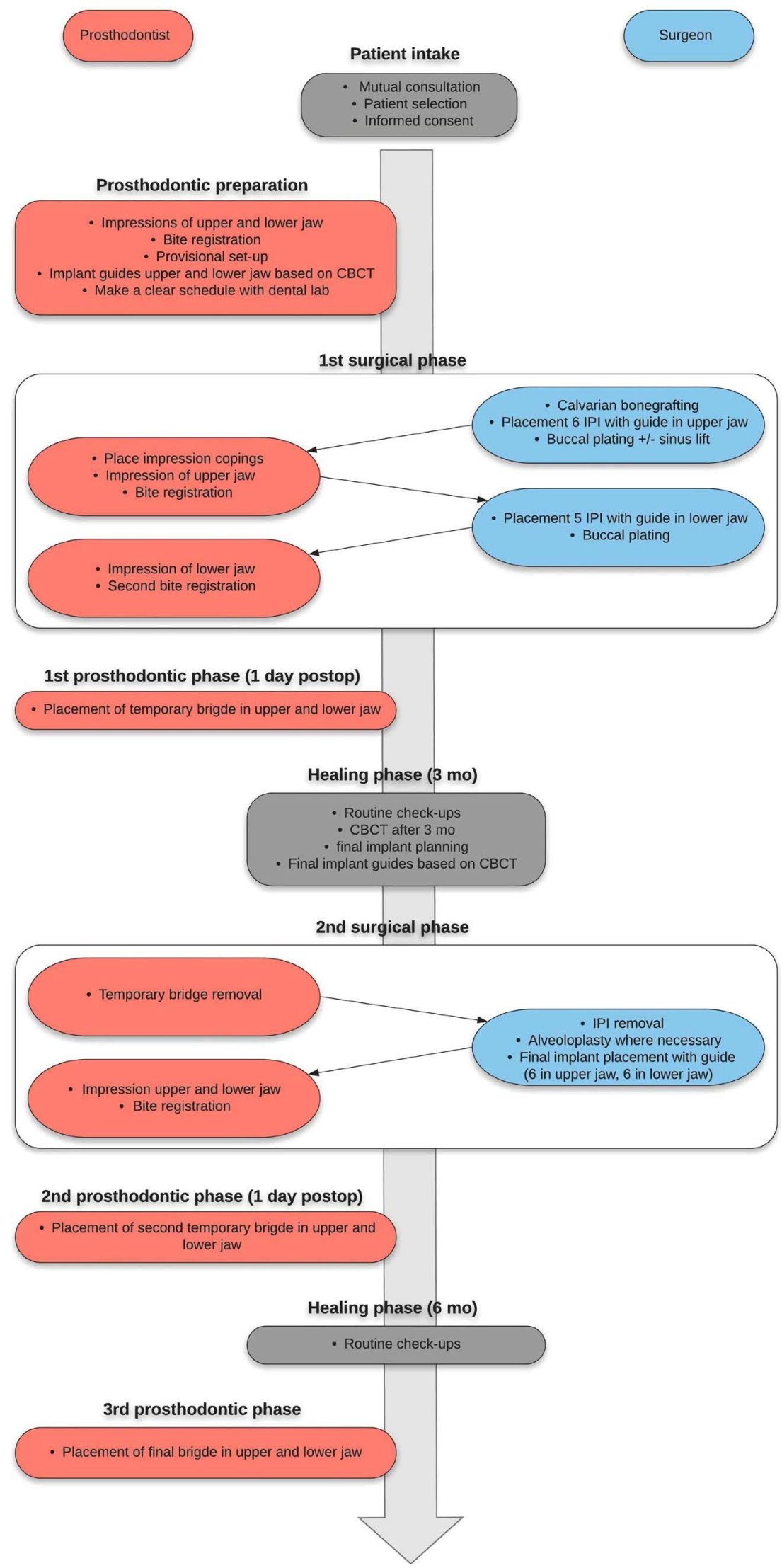

Figure 1. Flowchart of the complete surgical and prosthodontic process. 
this is in accordance with the patient's personal wishes. At the same time, a customised stereolithographic implant positioning template is fabricated based on the data of both preoperative CBCT and intraoral scan. This guide is designed in such a way that it indicates optimal IPI positioning but still allows for a sufficient degree of freedom. This feature is important since the condition of the native bone will always remain somewhat unpredictable (See Figure 2).

Preoperatively, a single dose of $1 \mathrm{~g}$ of cefazolin is given intravenously. After induction then, the first surgical phase (always performed by the same team of physicians) starts with calvarial bone harvesting. This is routinely performed using piezosurgical instruments at the parietal region of the patient's non-dominant side. Monocortical bone blocks are removed. After hemostasis, the contour of the vertex is restored by an injectable calciumphosphate cement (Hydroset, Stryker, USA). Closing of the wound is performed with resorbable sutures (Vicryl, Ethicon, Johnson\&Johnson, UK) and skin staples.

After bone harvesting, the surgery is continued intraorally with incision, periost slicing and atraumatic removal of the upper dentition (if still present). If necessary, conventional (bilateral) sinus lifting procedures are performed utilizing a lateral window approach. 6 IPIs (Nobel Biocare, Sweden) are placed in the upper jaw using the stereolithographic template. Since we plan to place the final implants at the site of the lateral incisor, first premolar and first molar, we place the IPIs at the central incisor, canine and second premolar locations. The intraoral parts of the IPIs are manipulated if necessary to achieve a parallel orientation. It is important to note that augmentation is only performed in areas where we plan to place an implant later on. This way we optimize the use of graft material to essential acceptor sites.

Buccal plating is carried out in concave regions of the upper jaw where necessary. The remaining bone is particulated using a bone mill, mixed with a bovine allograft (Bio-Oss, Geistlich Pharma, Switzerland) and applied to the sinus floor and on the buccal maxillary areas to fill out and smoothen the maxillary outline. A resorbable membrane (Ossix Plus, Memodent, the Netherlands) is used to cover the bone graft. The mucoperiosteum is meticulously closed using non- resorbable Ethilon sutures (Figure 3).

Next, the prosthodontist places impression copings on the IPIs in the upper jaw and takes an impression utilizing a rigid polyether (Impregum, 3M, Minnesota, USA). A bite registration is performed with a vinylpolysiloxane based material
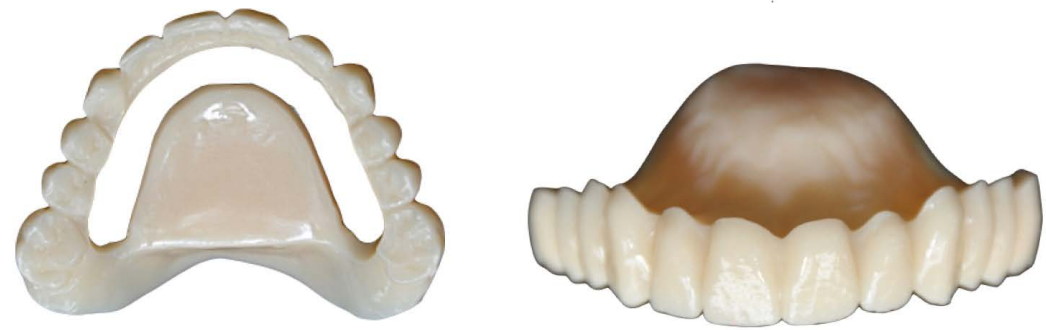

Figure 2. Photograph of a stereolithographical implant template. 


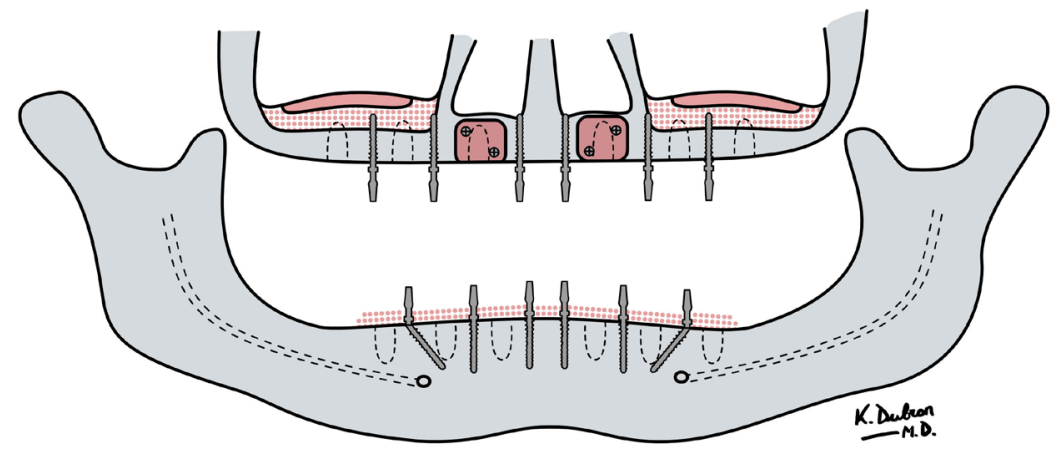

Figure 3. Schematic panoramic drawing providing an overview of location of IPIs and implants and location of bone grafts. Orange shapes represent the cortical bone grafts; dotted orange signifies granulated bone graft; dotted outlines indicate positions of final dental implants.

(Futar D, Kettenbach, Germany), using the lower jaw as reference for vertical height. The order of completing surgery and impression taking in the upper jaw first before moving on to the lower jaw, is of great importance. Using the lower jaw as a reference for occlusal height will ensure correct prosthetic rehabilitation in all further steps.

Finally, this protocol is repeated in the lower jaw where 5 IPIs (or in some cases 6 , when there is sufficient spacing available) are placed and buccal plating is carried out following the same technique.

The impressions are transported to the dental lab as soon as possible and temporary bridges are fabricated based on the wax-up which was previously fabricated. The next day, the temporary bridges are fitted and fixed after correcting occlusional contacts when necessary (Figures 4-6). The patient is then discharged with appropriate pain medication, chlorhexidine gel to apply around the IPIs and antibiotic therapy (amoxicillin and clavulanic acid $625 \mathrm{mg}, 3$ times daily for 7 days). Patients are advised to take some rest, avoid heavy lifting, use a soft diet and not blow their nose.

After ten days the patient is seen in the outpatient clinic to check healing progress, remove the staples from the donor site, remove intraoral sutures and check the function and occlusion of the provisional fixed bridges in both jaws.

After a healing period of 3 months, a second CBCT is made to check the quantity and position of the augmented bone. We strive to place implants (Astra Tech, Mölndal, Sweden) with a diameter of $4.2 \mathrm{~mm}$ and a length of $11 \mathrm{~mm}$. A new stereolithographic positioning template for the final implants is fabricated based on the data from this second CBCT.

During the second surgical phase, which is scheduled four months after the initial surgery, the provisional bridges are removed, mucoperiost flaps are raised and the IPIs are removed. Alveoloplasty is carried out where necessary. Then 6 final implants are placed in upper jaw (and 5 or 6 in the lower jaw), using the fabricated customised stereolithographic template. Again impressions are taken from upper and lower jaw and a bite registration is made. Here again, the same 

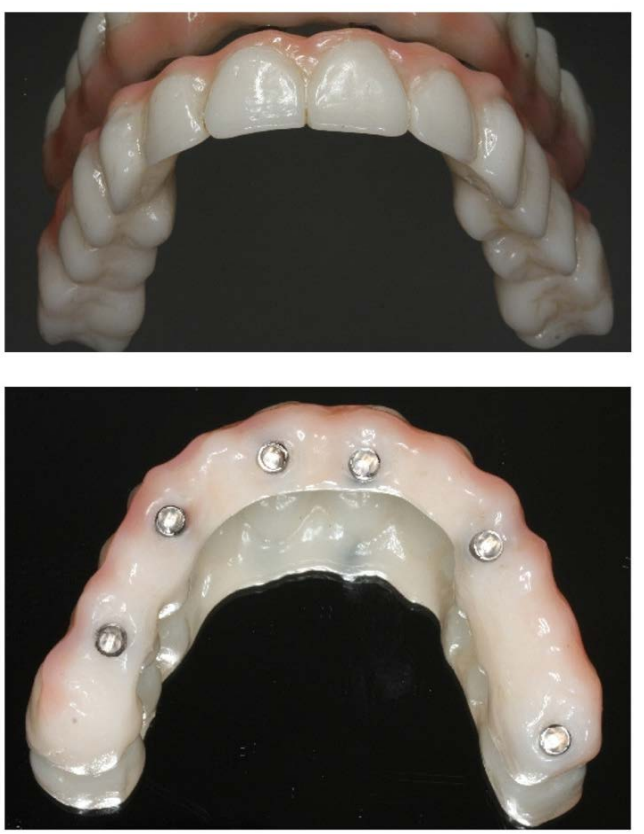

Figure 4. Photograph of the initial temporary upper bridge.
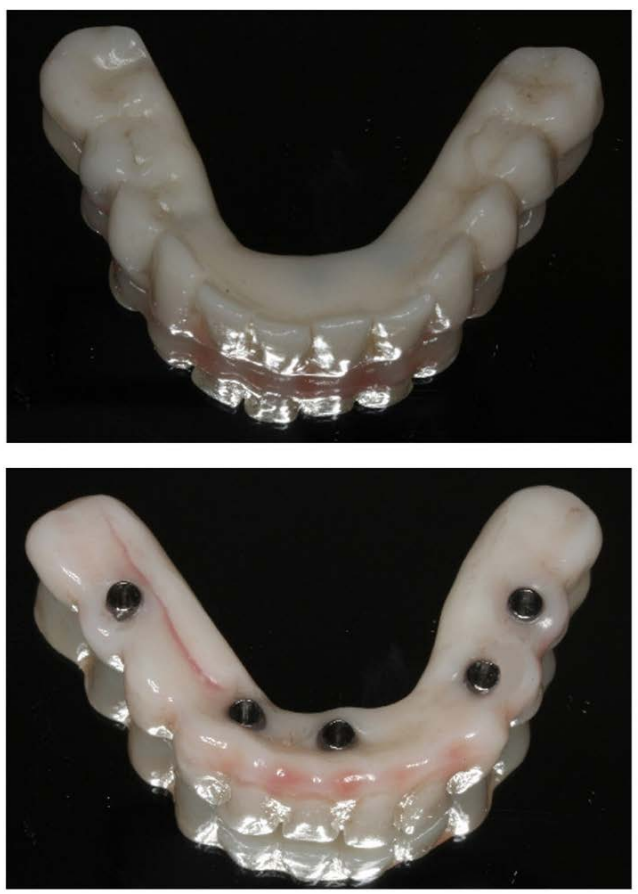

Figure 5. Photograph of the initial temporary lower bridge.
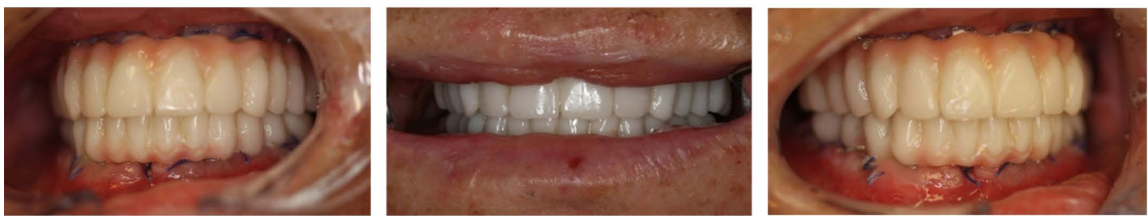

Figure 6. Clinical photograph after cementing of the temporary brigdes, 1 day after first stage surgery. Right view, front view, left view. 
step-by-step approach and sequence as in first stage surgery are used. Afterwards, an immediate loading protocol is followed, providing the patient with fixed provisional bridges on the final implants one day later. Ten days after discharge, the patient is recalled for the check-up of tissue healing and prosthodontic fine tuning. Recall at standardized intervals ensures patient compliance and good mucosal health. It also creates an opportunity to make some (aesthetic) corrections to the temporary restoration and reduces prosthetic problems. Figure 7 shows a radiographic overview of the process of one of the patients.

Four to six months after second stage surgery, the provisional bridges are replaced with final fixed bridges by the prosthodontist.

\section{Results}

Two women and one man with a mean age of 61 years (range 51 -74) were included in this case series. All patients went through the treatment plan as provided in the flowchart (Figure 1). A total of 33 IPIs were placed. All were immobile at second stage surgery. None displayed any type of infection. Intraorally, we did observe one dehiscence interforaminally at a region of buccal plating in the
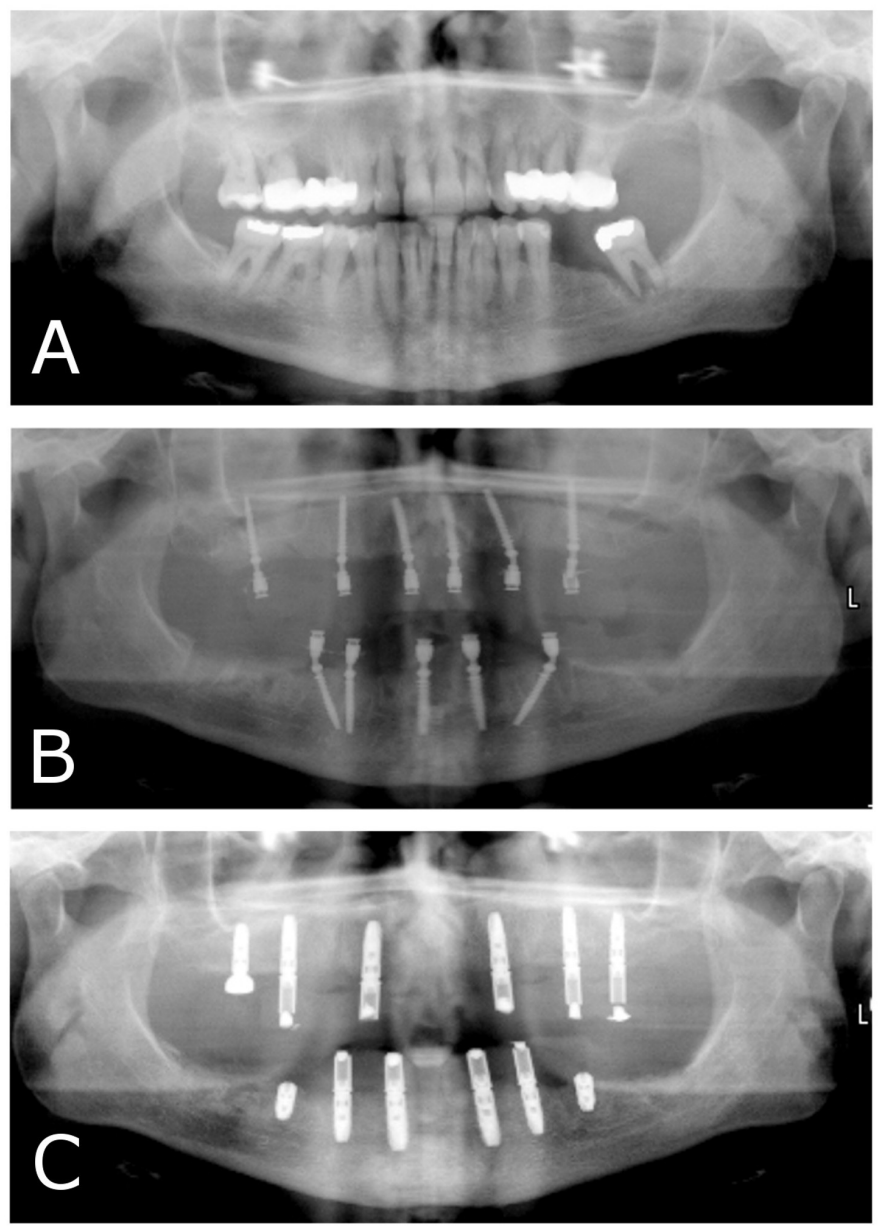

Figure 7. Panoramic radiograph (A) before treatment, (B) after first stage surgery and (C) after second stage surgery. 
male patient. No provisional bridges were lost and no infections were noted, so the success rate for the provisional bridges was $100 \%$. No other complications were observed.

At second stage surgery, a total of 12 dental implants were placed in each of the three patients: all had 6 implants placed in the upper jaw and 6 in the lower jaw. After a mean follow-up period of 25.67 months (20 - 29 months), none of these 36 implants were lost. There was some bone loss at one implant. In all patients, good functional and aesthetic results were obtained without any unforeseen complications. This renders the implant survival at $100 \%$ and the success rate at $97 \%$ using the modified success criteria described in Table 1.

\section{Discussion}

The use of implant retained dentures is a very common solution when conventional removable prostheses lack retention and stability or when the patient is adamant to have a more rigid oral rehabilitation option. Immediate implant placement after removal of remaining teeth may be an effective treatment for the latter [9] [10] [11]. When the alveolar ridge lacks sufficient volume however, immediate implant placement cannot be performed. Bone augmentation procedures will be required. These are routinely performed in common practice [12] [13]. The systematic review from Hämmerle et al. [14] shows that implant survival in regenerated bone versus non-regenerated bone is similar, making this a safe and predictable treatment option. However, graft healing and implant osseointegration do take up a significant amount of time. To avoid patient discomfort due to the ill-fitting prosthesis during this period, IPIs were introduced. These long and narrow screw-shaped dental implants can even be placed in severely atrophic jaws. The bendable transmucosal part can be manipulated in order for multiple IPIs to be parallel to each other and to serve as a proper base for immediate provisional prostheses. When properly carried out, immediate loading is a predictable option.

Lenssen et al. [1] combined these principles: during bone augmentation procedure, they fitted IPIs and followed an immediate loading protocol with a temporary fixed bridge. After graft healing, they removed the IPIs, placed the final dental implants and carried out immediate loading on these final implants as well.

The suitable patient population for this technique can be divided into two categories. The first are people who are edentulous and in need of a bone augmentation procedure followed by the implementation of an implant-supported structure but do not want to wait for 9 - 12 months. The second category consists of patients with a terminal dentition who have a high aesthetic and functional demand and strong desire for an immediate and fixed solution. Good patient selection is critical: patients need to be well informed and comply with the advice provided regarding perioperative medication, postoperative oral hygiene, etc. We also employ the help of an oral hygienist even if a periodontally com- 
prised dentition is present which is planned to be removed during surgery. Calculus is removed and the patient is taught proper oral hygiene. In our opinion, doing this in a preoperative phase ensures healthy gums at the time of surgery and a better patient compliance postoperatively. The patient should also be well informed about the costs and provided with a comprehensive informed consent.

In the described technique, we augment both upper and lower jaw simultaneously, remove any remaining teeth when necessary and place IPIs according to a strict protocol. During surgery, impressions are taken and the temporary bridge is fitted the next day. It must be noted that it is imperative that these IPIs are placed at the appropriate depth: the square plateau should be placed at the level of the crest (Figure 8). When placed too deeply, impressions will not be able to properly record enough details for accurate replication in the laboratory phase. This will lead to small deviations and result in an ill-fitting bridge. Placing the IPIs too superficially will possibly trap impression material underneath the screw head, making it very hard to remove the impression without distortion.

Regarding the positioning of the final dental implants, it is of great importance to pursue parallel placement for a screw-retained prosthetic design. This will avoid the need for angulated abutments which greatly facilitates the fabrication of a good fitting and easily placeable final implant bridge and will aid in proper maintenance. Moreover, as clearly shown in the article by Behnaz et al. [15], the splinting of implants placed parallel to each other produces the least amount of stress in the implant body and surrounding bone. This will ensure better osseointegration and implant success.

Looking onward, we see a possibility in completely digitalizing the prosthetic portion of the described technique. The knowledge, software and hardware required for a completely digital dental workflow are available. The application however remains a challenge to be met. Specifically, the swift intraoral scanning of implants in an edentulous jaw without any distortions may often still be the
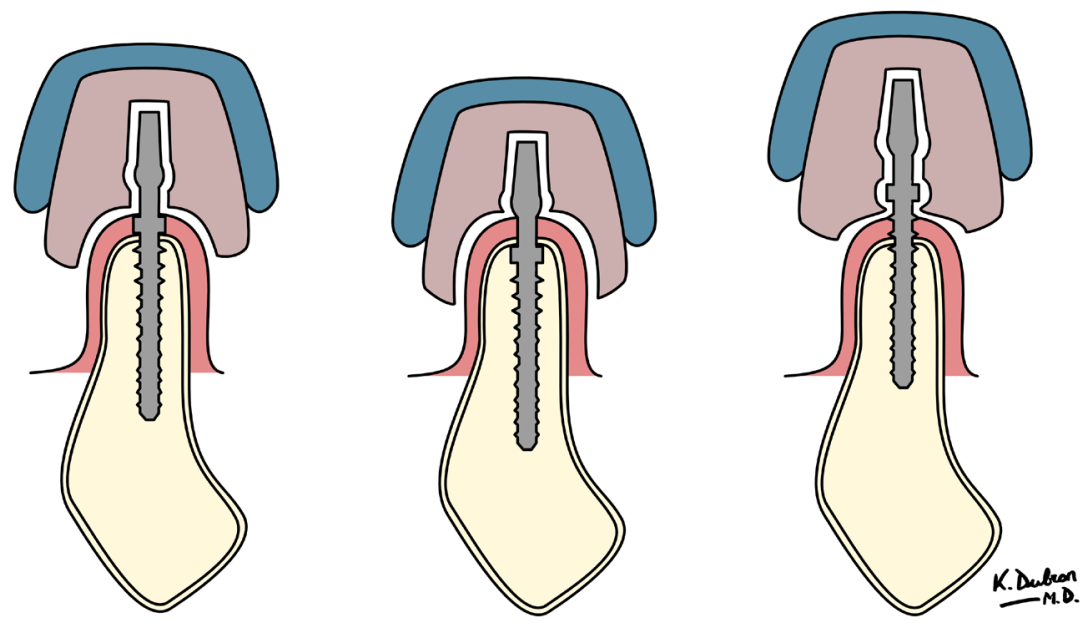

Figure 8. Cross sectional schematic representation of depth of insertion of IPIs and its implication with regards to impression taking. 
biggest hurdle to take.

The technique described in this article has several advantages. Firstly, the patient doesn't have to wait 9 - 12 months for a fixed solution. Masticatory function is restored immediately after surgery. There is no need to adapt an existing removable prosthesis, nor are there pressure points causing pain. And there is no other discomfort of wearing an ill-fitting prosthesis. This increases patient satisfaction greatly [1] [2] [16]. Secondly, the absence of a removable prosthesis pressing on the gums will avoid increased resorption of the graft during its healing period [1] [16]. Thirdly, the patient acquires experience with the sensation of wearing an implant-retained bridge. When the final bridge can be fabricated, the patient will have a realistic understanding of the design and function which will aid communication of any specific wishes to the prosthodontist.

As a downside, it should be noted that this procedure is complex, demanding a lot from each of the team members concerned. It should be noted that a very clear and efficient communication is needed with the dental lab ensuring a smooth workflow.

Although we did not observe mobile IPI's in any of the patients in het present study, mobility of IPI's is a common finding at second stage surgery. Most often this problem is not noted earlier because of the splinting of the IPIs. The sleek design with its aggressive thread and lead may produce a good primary stability. We hypothesize however that this specific design causes more localised ischemia in the bone surrounding the implant, causing increased peri-implant bone loss, possible increased risk for infectious problems and diminished long-term stability. This usually causes some granulomatous tissue to form at this site. Moreover, this complication may comprise later final implant placement. As a consequence, the final implant in this region will have to be placed in a slightly different location or a delayed loading protocol needs to be followed. To prevent this complication, we don't place IPIs in areas where we plan to place final implants later on. A final disadvantage is the increased cost for the patient caused by the use of IPIs and the temporary bridges. This is conveyed to the patient before treatment is initiated, so this is at the patient's discretion.

\section{Conclusion}

This study shows that the presented technique in this case series is a valid treatment option for patients who desire a fixed restoration, need pre-implantological bone augmentation procedures and do not want to function with a removable provisional prosthesis. Bone grafting is combined with placement of IPI in both upper and lower jaws with immediate provisional loading. After 4 - 5 months, the IPIs are removed and the final implants placed and a new immediate loading protocol is used. After osseointegration, final fixed bridges are fabricated. This technique is complex due to the intricate step-by-step process that is required and depends on a dedicated team to ensure a proper workflow. When these demands are met however, this procedure yields stable results with high patient sa- 
tisfaction.

\section{Acknowledgements}

We wish to express our sincere gratitude to Kathia Dubron (MD) for creating the marvellous drawings published in this article.

Secondly, many thanks go out to the dental laboratory Schouten in Waalwijk for their continuing innovation, ability to meet our high expectations and willingness to weigh in on complex cases.

\section{Conflicts of Interest}

The authors declare no conflicts of interest regarding the publication of this paper.

\section{References}

[1] Lenssen, O., Barbier, L. and De Clercq, C. (2011) Immediate Functional Loading of Provisional Implants in the Reconstructed Atrophic Maxilla: Preliminary Results of a Prospective Study after 6 Months of Loading with a Provisional Bridge. International Journal of Oral and Maxillofacial Surgery, 40, 907-915. https://doi.org/10.1016/j.ijom.2011.05.001

[2] Krennmair, G., Krainhöfner, M., Weinländer, M. and Piehslinger, E. (2008) Provisional Implants for Immediate Restoration of Partially Edentulous Jaws: A Clinical Study. International Journal of Oral and Maxillofacial Implants, 23, 717-725.

[3] Barbier, L., Abeloos, J., De Clercq, C. and Jacobs, R. (2012) Peri-Implant Bone Changes following Tooth Extraction, Immediate Placement and Loading of Implants in the Edentulous Maxilla. Clinical Oral Investigations, 16, 1061-1070. https://doi.org/10.1007/s00784-011-0617-9

[4] Gagnier, J., Kienle, G., Altman, D., Moher, D., Sox, H. and Riley, D. (2013) The CARE Guidelines: Consensus-Based Clinical Case Reporting Guideline Development. Journal of Medical Case Reports, 7, Article No. 223. https://doi.org/10.1186/1752-1947-7-223

[5] Albrektsson, T., Zarb, G., Worthington, P. and Eriksson, A. (1986) The Long-Term Efficacy of Currently Used Dental Implants: A Review and Proposed Criteria of Success. International Journal of Oral and Maxillofacial Surgery, 1, 11-25.

[6] Needleman, I., Chin, S., O’Brien, T., Petrie, A. and Donos, N. (2012) Systematic Review of Outcome Measurements and Reference Group(s) to Evaluate and Compare Implant Success and Failure. Journal of Clinical Periodontology, 39, 122-132. https://doi.org/10.1111/j.1600-051X.2011.01836.x

[7] Papspyridakos, P., Chen, C.-J., Singh, M., Weber, H.-P. and Gallucci, G. (2012) Success Criteria in Implant Dentistry: A Systematic Review. Journal of Dental Research, 91, 242-248. https://doi.org/10.1177/0022034511431252

[8] Cawood, J. and Howell, R. (1988) A Classification of the Edentulous Jaw. International Journal of Oral and Maxillofacial Surgery, 17, 232-236. https://doi.org/10.1016/S0901-5027(88)80047-X

[9] Botticelli, D., Persson, L., Lindhe, J. and Berglundh, T. (2006) Bone Tissue Formation Adjacent to Implants Placed in Fresh Extraction Sockets: An Experimentale Study in Dogs. Clinical Oral Implant Research, 17, 351-358. https://doi.org/10.1111/j.1600-0501.2006.01270.x 
[10] Araújo, M. and Lindhe, J. (2005) Dimensional Ridge Alterations following Tooth Extraction. An Experimental Study in the Dog. Journal of Clinical Periodontology, 32, 212-218. https://doi.org/10.1111/j.1600-051X.2005.00642.x

[11] Araújo, M., Sukekava, F., Wennström, J. and Lindhe, J. (2006) Tissue Modeling Following Implant Placement in Fresh Extraction Sockets. Clinical Oral Implant Research, 17, 615-624. https://doi.org/10.1111/j.1600-0501.2006.01317.x

[12] Nyström, E., Nilson, H., Gunne, J. and Lundgren, S. (2009) A 9-14 Year Follow-Up of Onlay Bone Grafting in the Atrophic Maxilla. International Journal of Oral and Maxillofacial Surgery, 38, 111-116. https://doi.org/10.1016/j.ijom.2008.10.008

[13] Tessier, P., Kawamoto, H., Matthews, D., Posnick, J., Raulo, Y., Tulasne, J. and Wolfe, S. (2005) Autogenous Bone Grafts and Bone Substitues-Tools and Techniques: I. A 20,000-Case Experience in Maxillofacial and Craniofacial Surgery. Plastic and Reconstructive Surgery, 116, 6S-24S.

https://doi.org/10.1097/01.prs.0000173862.20563.12

[14] Hämmerle, C., Jung, R. and Feloutzis, A. (2002) A Systematic Review of the Survival of Implants in Bone Sites Augmented with Barrier Membranes (Guided Bone Regeneration) in Partially Edentulous Patients. Journal of Clinical Periodontology, 29, 226-231. https://doi.org/10.1034/j.1600-051X.29.s3.14.x

[15] Behnaz, E., Ramin, M., Abbasi, S., Pouya, M. and Mahmood, F. (2015) The Effect of Implant Angulation and Splinting on Stress Distribution in Implant Body and Supporting Bone: A Finite Element Analysis. European Journal of Dentisty, 9, 311-318. https://doi.org/10.4103/1305-7456.163235

[16] Östman, P.-Ö., Hellman, M., Nilson, H. and Ericsson, I. (2004) Provisional Implants: A Clinical Prospective Study in 45 Patients, from Implant Placement to Delivery of the Final Bridge. Clinical Implant Dentistry and Related Research, 6, 142-149. 